\title{
IDENTIFICATION OF GAPS IN SPATIAL AWARENESS DEVELOPMENT TESTS AND MEANS OF THEIR ELIMINATION
}

\author{
Victoria Honta \\ Department of descriptive geometry and engineering graphics \\ Kyiv National University of Construction and Architecture \\ 31 Povitroflotsky ave., Kyiv, Ukraine, 03037 \\ victoriahonta@gmail.com
}

\begin{abstract}
The article examines the existing methods for checking spatial awareness on the basis of which seven of the most common defects of test content are detected, which automatically grow into a gap in the spatial awareness development tests (SADT). The work describes ways to minimize the effect of gaps in the test, or complete elimination by the proposed and proven methods. There was found the essence of the analogy of various SADT and the direct application of general and innovative testing principles as means of overcoming the gaps. The cases, where overcoming of the gaps is the basis for the development of spatial awareness and spatial thinking, were identified. There was systematically reviewed the module of unprocessed, missed tasks in the test, which led to a detailed analysis and investigation of the representation of graphic material in the tasks. Mini-testing of the influence of the graphic component on the speed and quality of the accomplishment of spatial tasks was carried out. The results of the mini-test showed the smallest number of errors in the cubic traditional model and the traditional model with use of shadows, indicating their successful use in the created training and evaluation course "PROSTIR-UA". Also, there was illustrated the necessity after processing of the test results, there was presented an example of the use of a block diagram of a combined algorithm of the spatial awareness development test, in the final stage of which, for the test taker, a recommendation base of the necessary auxiliary tasks is made, based on the results of the testing. The presented study aims to offer advanced approaches to existing methods and methods of motivating students to effectively use spatial awareness development tests and new tools for expanding and applying spatial knowledge.
\end{abstract}

Keywords: spatial awareness, gaps in SADT, spatial thinking, flaw of classical testing, graphic tools.

\section{Introduction}

There are no perfect tests for the development of spatial awareness. Modern tests like all other tests do not lack the gaps that occur, in particular, due to change, upgrading, improvements and many other reasons. The gaps exist in testing and they will be even in the most stable and innovative spatial tests, since it is objectively impossible to predict all situations that will require system regulation in the future.

It is no coincidence that in 2018, scientists from the University of Salzburg, Guenter Maresch and Theresa Pomberger, emphasized the need of the consideration of the problems of spatial awareness development tests $[1,2]$. The focus was on reviewing the results of gender differences in spatial perception, there were discussed the results connected with the possible consequences of gender differences and the effects of adaptation. A number of studies on the GeodiKon project were also conducted, one of its sections was focused on the conclusions of the use of various perspective strategies for solving spatial problems, connection between sports and spatial awareness [3]. The experiments that were carried out are a continuation of efforts in the direction of expanding spatial problems and the development of spatial awareness methods in general.

Planning of new methods of assessing spatial thinking skills requires a thorough study of existing techniques, understanding spatial skills and the availability of means for evaluating them during their application. The potential significance of learning and developing spatial thinking and awareness in children can be found in works written by Zachary Hawes, Joan Moss, Beverly Caswell, Sarah Naqvi, Sharla MacKinnon, Andrea Frick, Nora Newcombe [4, 5]. It is necessary to pay special attention to the method "Mental Folding Test for Children" that is developed for children, who are 4-7 years old, the results of the test indicate the need for gradual complication of tasks and indicate the need to provide the proposed mean in preschool and elementary school [6]. 
Further research into spatial awareness should further determine the use of qualitative tools, clearly defined organizational moments for testing (submission), preliminary identification of clear relation between the test taker's tasks and responses, and should be used by persons, who are over 14 , this will allow tracking the development level of spatial awareness at each stage of the course, regardless of the gained experience.

Given that tests as well as the right "should not be reduced to standards only" [7], our goal is to investigate the gaps in the form of presentation of the test, its accomplishment and after error handling. It should be noted, that the gap in the SADT cannot be identified with "error or defect" as the result of an incorrect assessment of objective conditions, the presentation and manifestation of the problem of estimating spatial awareness and thinking.

\section{Aim of research}

The purpose of the consideration of this problem is to determine the possibilities of improving the incompleteness of the content base and the structure of the algorithm for creating improved tests for the development of spatial imagination.

\section{Materials and methods of research}

The methodological basis of the research is the dialectical method, survey technique and mini-testing. During the survey and mini-testing, general scientific and special methods were used, among them the most significant are: abstract-logical method for the formulation of the research findings; methods of analysis, synthesis and generalization for studying the features of SADT creation.

\section{The results of the work}

The gap in the SADT, as a rule, occurs as a result of the fact that during the process of passing the test there were not foreseen organizational nuances or considered system support for the development of spatial awareness outside the scope of testing or during its passing. Thus, the gap can be considered incomplete in the content. During the study, only those nuances that can be resolved or improved with the help of additional tasks [8] or the training instructions for the accomplishment of the tasks are taken into account.

\section{The most common gaps in SADT were identified:}

1. 1. Introductory tutorial with use of the graphic tools

The introductory briefing is one of the most effective types of training in the matter of correct accomplishment of the works. By the nature and time of this briefing, it can be called introductory and target-oriented. The tutorial can be individual or group, but it must be graphical, simple, and understandable to the user. During the creation of the training and evaluation base "PROSTIR-UA" there was used the remote platform Moodle, which allows creating an interesting and accessible introductory tutorial with the implementation of the graphic tools.

\section{2. Missed tasks in the test that were not worked out}

Missed tasks in the test may indicate:

- incomprehensibility of the objective;

- not found a final solution of the problem;

- uncertainty in the choice or solution of the task;

- negligent attitude towards testing.

During the creation of the training and evaluation base "PROSTIR-UA", a lot of attention was paid to the clear verbal and graphical representation of the tasks and created spatial awareness tests. If the verbal job setup is completely obvious, it is possible to experiment with the graphic component of the problem.

The issue of various forms of presentation of the graphic component of the problem was raised. Such forms of presentation can be:

- traditional testing model MRT (Fig. 1) [9];

- traditional model with use of shadows (Fig. 2); 
- model with color blocks (Fig. 3). For the third form, different segments are represented, that is, a combination of several colorful cubes;

- realistic model (Fig. 4). The purpose of the fourth form of submission was to present objects as realistically as possible. In order to do that, a light source was exposed to create a shadow and one color was selected, which reproduces the plastic.

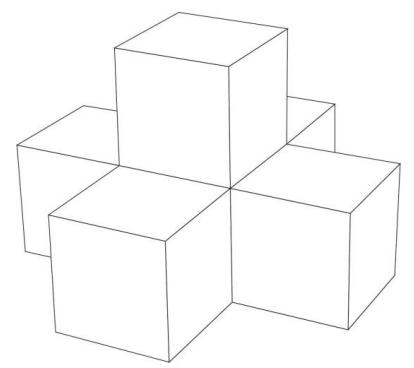

Fig. 1. Traditional model of MRT testing

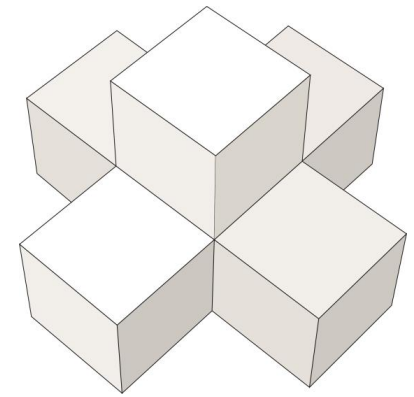

Fig. 2. Traditional model of MRT testing

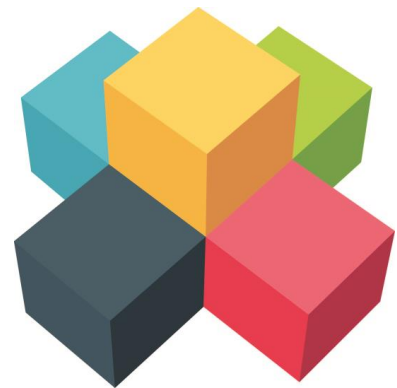

Fig. 3. Model with color blocks

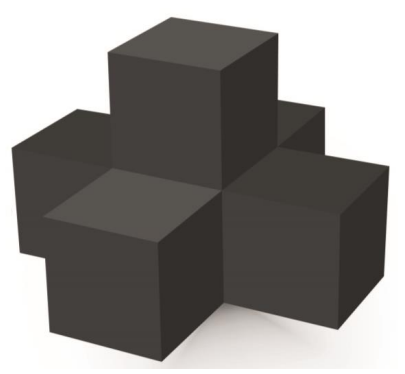

Fig. 4. Model with realistic blocks

Maximum comprehension was achieved through the use of an object consisting of five cubes and it is done in four different forms of the previously presented submission. 
Thus, having several forms of representation of the graphic base of the task, a survey and mini-testing were conducted to get to know which of the forms of representation of graphic information is the most acceptable and understandable for ordinary citizens of different professions and ages. 10 people participated in the survey and mini-testing.

Previously, it was clearly stated that it was a matter of spatial awareness and the tasks did not have the desired thinking process, but everyone could use their own strategy for solving the tasks. Each test taker was given 4 tasks of the same complexity but with different graphical representation of the information. Each task is placed separately from others on the sheets of colored paper. The test takers are warned that the tasks can be done in any sequence, at their discretion, it does not affect the final result, but after the mini-testing it was discovered that most of the test takers (8 out of 10) chose a task with a traditional shadow image, as it depicts a figure with fewer "stones". However, since this task was chosen first, the testers needed more time to study the issue, so this is not a significant difference among the tasks. Also, the test takers were warned that for doing each task they had 2 minutes (Fig. 5, Table 1).
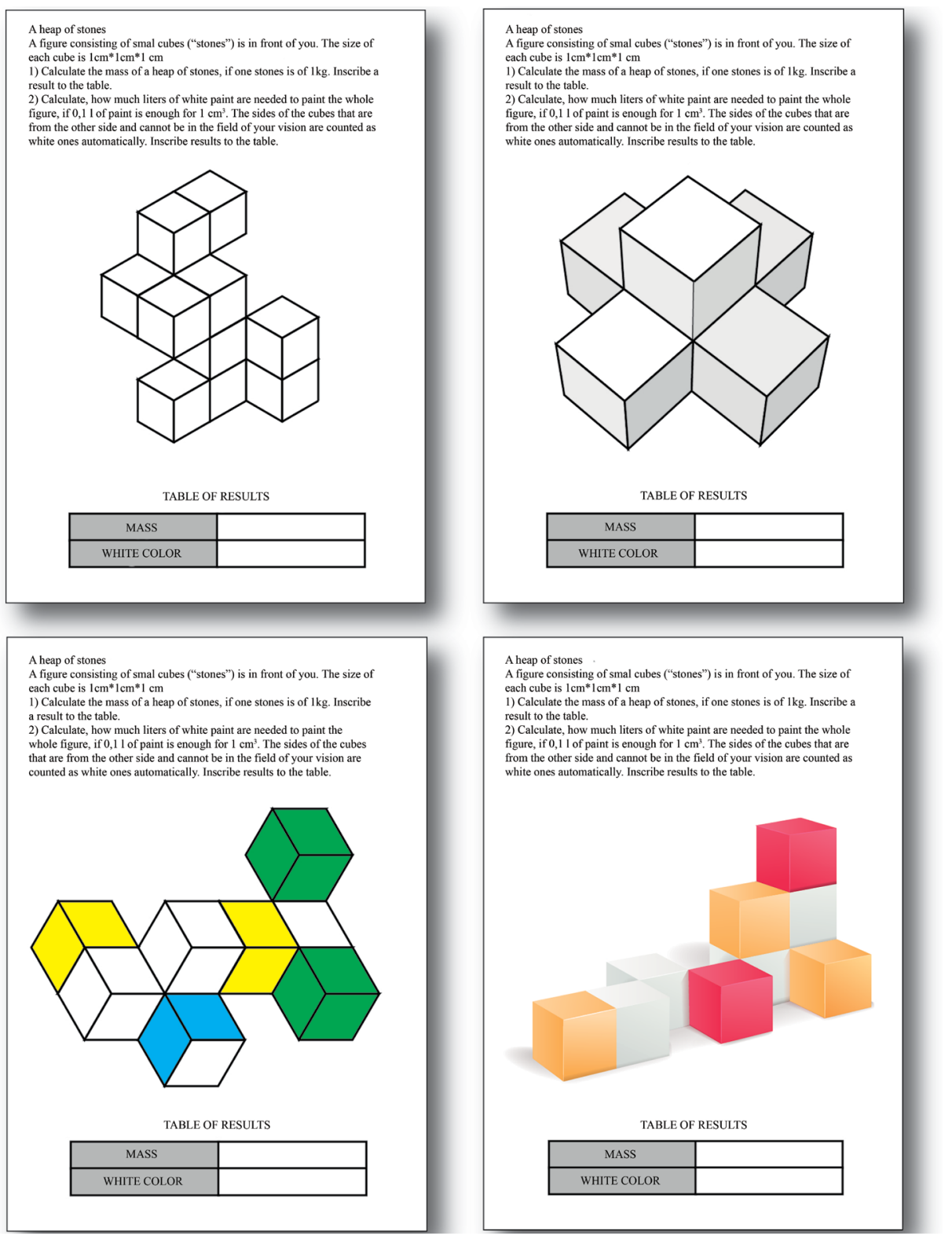

Fig. 5. Task of the mini-testing with different presentation of the information 
The results, presented in Table 1, showed that the least mistakes were made in the cubic traditional model and the traditional model using shadows, followed by a model with color blocks. Most of the mistakes and additional questions were made in the realistic model.

Table 1

Distribution of presentation forms to various tasks

\begin{tabular}{|c|c|c|c|c|c|c|c|c|c|c|c|}
\hline \multirow{2}{*}{ Display format } & \multicolumn{10}{|c|}{ Test takers' answers } & \multirow{2}{*}{$\begin{array}{c}\text { Average } \\
\text { time }\end{array}$} \\
\hline & 1 & 2 & 3 & 4 & 5 & 6 & 7 & 8 & 9 & 10 & \\
\hline Traditional & 1 & 1 & 1 & 1 & 1 & & 1 & 1 & 1 & 1 & 1,30 \\
\hline Traditional with shadow & 1 & 1 & 1 & 1 & 1 & 1 & 1 & 1 & 1 & 1 & 1,45 \\
\hline Color model & 1 & 1 & & 1 & 1 & & 1 & & 1 & 1 & 1,50 \\
\hline Realistic model & 1 & 1 & 1 & 1 & & & & 1 & 1 & & 1,55 \\
\hline Result & 4 & 4 & 3 & 4 & 3 & 1 & 3 & 3 & 4 & 3 & \\
\hline
\end{tabular}

1. 3. Partly not done or incorrectly done tasks in the test can indicate the same reasons as missing the tasks, but to this list it is possible to add an inattentive studying of the task or accomplishment.

1. 4. Test taker's incorrect answers can be accompanied by the problems, mentioned in the paragraphs 1.2. and 1.3, it is also possible to add a complete misunderstanding of the task.

1. 5. Unintentional or unconscious concealment of the test results for the test taker.

In this case, it is proposed to consider the problem of the hidden testing, when test results are reported to the test taker in the form of evaluation or points, but herewith paragraphs 1.2, 1.3, and 1.4 are not considered and studied. Such testing is ineffective, especially if it concerns SADT. Such tests are aimed not only at checking the test taker's spatial knowledge and skills, but also at the process of learning and extraction of new spatial information, which can be applied not only during the training but also in life.

1. 6. Error correction with use of the graphic tools

A variety of error correction activates the test taker's cognitive activity, causes and retains a cognitive interest in obtaining new knowledge. This allows to discover one's capabilities and spatial abilities in the best possible way. It is precisely this point of the SADT's gaps that is the most widespread and the least studied.

Consequently, the main step in eliminating the gaps in the organization of improving the testing of the ADT is not the testing itself but its following processing. The use of the productive block diagram of the combined algorithm of SADT [8] on the training and evaluation basis "PROSTIR-UA" makes it possible to use an additional function after processing. Upon completion of the basic testing and evaluation, a message appears on the screen recounting all the test taker's false responses, further offering their detailed consideration and a step-by-step solution to similar types of tasks with use of a variety of the graphical tools that will improve the perception of new information and visual memorization.

1. 7. Repeated effective accomplishment of unfulfilled tasks for fixing the material.

However, even after the detailed review and step-by-step solution to similar tasks to the previously unresolved ones, it is obligatory to re-pass the main full-test. This will allow to identify all the hidden moments, for example, if the test taker chooses the answer at random and this answer is correct, it is unlikely that he chooses the same answer a second time, then he/she will need to go through the educational step-by-step solution of the tasks that have similar types to be able to go through the second level, more complex test, which is closed until the test taker completes the previous test successfully.

\section{Discussion of the results of the research}

These studies have shown that most of the gaps in the spatial awareness tests are laid down during testing, organizing (or submitting) the test and after using it. This indicates a positive change 
in the attitude towards the previously proposed block diagram of the combined algorithm of spatial awareness development tests [8] and confirms Harris, J., Hirsh-Pasek, K., Newcombe, N. S.'s study on the need for gradual complication of tasks [10].

We also want to note the difference between the results of the research of the influence of the graphical representation of the information in the testing tasks with the results of Guenter Maresch, Karl Josef Fuchs, Simon Plangg, Marion Zöggeler [10], however, we note that we did not take into account gender specificity, and the age of the participants of the mini-testing exceeded 18 years, Also, a function of time was used, which could significantly affect the research results.

The use of the testing as the tool of assessment requires from a teacher knowledge of the theory of testing, skills and experience in analyzing the results and assessing the test takers' achievements. Until testing as a tool for measuring the progress and effectiveness of the spatial awareness development process is not worked out and understood well, the test will not be qualitative.

The idea of creating a training and evaluation base "PROSTIR-UA" consists in desire to maximize the results of the development of spatial awareness in the test takers, taking into account the maximum number of significant nuances, proved by the scientific research.

The main advantage of this study is the attempt to identify the gaps in the spatial awareness development tests with further elimination of them by the previously proposed methods. Creating a "PROSTIR-UA" database will meet the priorities of the development of modern education, research and innovation, as well as improve the role of spatial awareness tests as an instrument for enhancing the various fields of education.

This study is useful for efficient planning and the creation of SADT. This research is a continuation of the author's previous works, therefore, it is planned to keep working on the topic and its continuation.

\section{Conclusion}

1. The important issues concerning the analysis of the development of spatial awareness development tests and finding gaps at different stages of testing are highlighted, this is an important prerequisite for creating a more efficient database that can provide a higher level of spatial awareness verification as well as its practical application for effective execution of diverse spatial problems.

2. Test control can be used to actualize the test-takers' spatial knowledge and skills, estimate the levels of the academic progress of the academic groups and individuals, analyze various forms and methods of training and for the final evaluation of the control. However, it should be remembered, that spatial test control should be conditioned primarily by the features of the subject and the test taker's peculiarities. Therefore, it is necessary to prepare gradually the test takers for such a form of control, and after passing it, it is necessary to work out all the mistakes made by the testers taking into account time and other features.

3. Having reviewed the gaps in the SADT more broadly and dividing them into separate elements, we can state with certainty the fact that they can be improved or even overcome. Contrary to the hypothesis that the presentation does not affect the results of the spatial awareness or thinking test, it is possible to state that the graphical representation of the information in the task affects the measurement of the timeline and the test takers' mental capabilities. If one follows pre-recommended ways to solve the gaps in future work with SADT, it will bring an excellent result in creating perfect testing and improving spatial skills.

\section{References}

[1] Pomberger, T., Maresch, G. (2018). Geschlechterspezifische Unterschiede beim Raumvorstellungsvermögen. Mathematik im Unterricht, 9, 63-74.

[2] Maresch, G., Scheiber, K. (2017). Practical and Structured Exercises to Train Geometry and Your Spatial Ability, 54. 
[3] Herbst, P., Cheah, U. H., Richard, P. R., Jones, K. (Eds.) (2018). International Perspectives on the Teaching and Learning of Geometry in Secondary Schools. Springer International Publishing, VI, 386. doi: http://doi.org/10.1007/978-3-319-77476-3

[4] Hawes, Z., Moss, J., Caswell, B., Naqvi, S., MacKinnon, S. (2017). Enhancing Children’s Spatial and Numerical Skills through a Dynamic Spatial Approach to Early Geometry Instruction: Effects of a 32-Week Intervention. Cognition and Instruction, 35 (3), 236-264. doi: http://doi.org/10.1080/07370008.201 7.1323902

[5] Frick, A., Newcombe, N. S. (2012). Getting the big picture: Development of spatial scaling abilities. Cognitive Development, 27 (3), 270-282. doi: http://doi.org/10.1016/j.cogdev.2012.05.004

[6] Harris, J., Newcombe, N. S., Hirsh-Pasek, K. (2013). A New Twist on Studying the Development of Dynamic Spatial Transformations: Mental Paper Folding in Young Children. Mind, Brain, and Education, 7 (1), 49-55. doi: http://doi.org/10.1111/mbe.12007

[7] Kelman, M. S., Murashyn, O. H. (2008). Zahalna teoriia derzhavy i prava. Kyiv: Kondor, 477.

[8] Honta, V. (2015). Algorithmization tests development Algorithmization tests development orithmization tests development of spatial imagination. Motrol. Commission of motorization and energetics in agriculture, 17 (8), 61-66

[9] Peters, M., Laeng, B., Latham, K., Jackson, M., Zaiyouna, R., Richardson, C. (1995). A Redrawn Vandenberg and Kuse Mental Rotations Test - Different Versions and Factors That Affect Performance. Brain and Cognition, 28 (1), 39-58. doi: http://doi.org/10.1006/brcg.1995.1032

[10] Maresch, G., Fuchs, K. J., Plangg, S., Zöggeler, M. (2018). Wirkung unterschiedlicher Darstellungsformen bei Aufgaben zur mentalen Rotation. Mathematik im Unterricht, 9, 75.

\title{
NOMADISM AS A WAY OF BEING OF THE IMMIGRANTS AND INTERNALLY DISPLACED PERSONS
}

\author{
Marina Kolinko \\ Department of Philosophy \\ Vasyl Stus Donetsk National University \\ 21 600-richya str., Vinnytsia, Ukraine, 21021 \\ mkolinko09@gmail.com
}

\begin{abstract}
The article presents the innovatory understanding of the nomadic strategy of human being in the transitional condition. The aim of the article is to determine the role of the nomadic being way in the social group of internal migrants. It is substantiated, that aims and actions of a nomad are directed on creating new ways of realization and conceptualization of variants of nomadic being. It is explained, that a nomad doesn't go by the way, offered by traditional types of activity, but searches innovatory ways of realization, doesn't stop on deciphering of traditionally existing being senses, but produces them him/herself. A subject, living in a space of "boundaries" is deprived of the settled comfort, he/she searches for a possibility of balance and harmony, social recognition and improvement of own life conditions in the movement and change. The culture of choice, formed by the logic of the modern market, gives a nomad resources for regulating the own freedom degree. Nomadic instruments correspond to the migrants' way of life. Digital nomadism creates stimuli for the active life and adaptation to new conditions of different groups of migrants.
\end{abstract}

Keywords: internally displaced persons (IDP), immigrants, boundary, nomadism, digital nomad.

\section{Introduction}

Among topical, acute social problems that need philosophical understanding, a leading place is occupied by the theme of transformation of socio-cultural interactions in the dynamically changeable world. "Boundary" challenges of today explain the interest to anthropological and socio-cultural aspects of the theme of boundary and transitional condition of being, caused by migration processes, wars, political and religious events in the world. Such challenges include military 